\title{
A Genetic Algorithm for Locating the Multiscale Critical Slip Surface in Jointed Rock Mass Slopes
}

\author{
Qiang Xu, ${ }^{1}$ Jian-yun Chen, ${ }^{1,2}$ Jing $L i,{ }^{1}$ and Hong-yuan Yue ${ }^{1}$ \\ ${ }^{1}$ School of Civil and Hydraulic Engineering, Dalian University of Technology, Dalian 116023, China \\ ${ }^{2}$ State Key Laboratory of Coastal and Offshore Engineering, Dalian University of Technology, Dalian 116023, China \\ Correspondence should be addressed to Qiang Xu; xuqiang528826@163.com
}

Received 17 September 2013; Revised 23 December 2013; Accepted 31 December 2013; Published 12 February 2014

Academic Editor: Jyh-Horng Chou

Copyright (c) 2014 Qiang Xu et al. This is an open access article distributed under the Creative Commons Attribution License, which permits unrestricted use, distribution, and reproduction in any medium, provided the original work is properly cited.

\begin{abstract}
The joints have great influence on the strength of jointed rock mass and lead to the multiscale, nonhomogeneous, and anisotropic characteristics. In order to consider these effects, a new model based on a genetic algorithm is proposed for locating the critical slip surface (CSS) in jointed rock mass slope (JRMS) from its stress field. A finite element method (FEM) was employed to analyze the stress field. A method of calculating the mechanical persistence ratio (MPR) was used. The calculated multiscale and anisotropic characteristics of the MPR were used in the fitness function of genetic algorithm (GA) to calculate the factor of safety. The GA was used to solve optimization problems of JRMS stability. Some numerical examples were given. The results show that the multiscale and anisotropic characteristics of the MPR played an important role in locating the CSS in JRMS. The proposed model calculated the CSS and the factor of safety of the slope with satisfactory precision.
\end{abstract}

\section{Introduction}

Slope stability analysis is important for assessing the safety of earth dams and natural slopes. However, stability analysis of JRMS is one of the important problems for geological engineering. Locating the CSS of JRMS is the chief concern. Because the rock masses consist of a large number of joints, they have the multiscale, nonhomogeneous, and anisotropic material characteristics. These reasons lead to the complex of deformation and stress distributions in JRMS and make the analysis of JRMS difficult. As a powerful tool, GAs are used widely in the homogeneous slope stability analysis. Goh [1] employed a GA to search for the minimum factor of safety of homogeneous slope. Mccombie and Wilkinson [2] presented a simple GA to locate the CSS of a homogeneous layered slope. Zolfaghari et al. [3] proposed a simple GA to locate the critical noncircular failure surface of a homogeneous layered slope. Sun et al. [4] applied a spline curve in conjunction with a GA to locate the CSS in slope stability analysis. Sengupta and Upadhyay [5] presented a GA to search for the minimum factor of safety of a homogeneous slope. Li et al. [6] used a real coded GA to search for the noncircular CSS of a homogeneous layered slope. However, these methods cannot solve such problems in nonhomogeneous and anisotropic material. FEM, on the other hand, has been used for the analysis of slope stability. In contrast to the simplified techniques, FEM can solve the problem of the growth of inelastic zones with time and a complex loading sequence. Some tests have to be carried out according to the concept and application. Kim and Lee [7] used FEM to locate the CSS of a slope. Cho and Lee [8] proposed a FE flow-deformation coupled analysis program for solving unsaturated slope stability problems. Wang et al. [9] employed GA and FEM to locate the critical circular slip surface. Zheng et al. [10] used FEM and strength reduction techniques to calculate the safety factors for slopes. Others of researchers focused on the influence of joints on the material strength of jointed rock mass. Du et al. [11] analyzed the mutual influences of joints and rock bridges. Chen et al. [12] studied the influence of joints on the shape of the CSS in JRMS and described the shape of CSS in JRMS as noncircular. Zhang et al. [13] used the minimum joints frequency to locate the CSS of JRMS. 
In this paper, based on the recent studies, a new model based on GA was proposed to search for the CSS in JRMS from its stress field. The studies on the influences of joints on multiscale and nonhomogeneous characteristics of jointed rock masses were made. The methods for calculating the factor of safety of slope based on the stress field, as calculated by FEM, were proposed. Then the GA was used to locate the CSS of JRMS. And then the numerical examples were analyzed to verify the proposed model.

\section{The Algorithm for Locating the Multiscale CSS}

2.1. The Factor of Safety in the Method of Slices. The factor of safety $F$ in the method of slices is expressed as

$$
F=\frac{\int_{\Gamma} \tau_{f} d \Gamma}{\int_{\Gamma} \tau_{n} d \Gamma},
$$

where $\tau_{n}$ is the mobilized shear stress at any point along the slip surface, $\tau_{f}$ is the shear strength at the corresponding point, and $\Gamma$ is the length of the slip surface.

The mobilized shear stress $\tau_{n}$ and the shear strength $\tau_{f}$ can be calculated from the stress field which has been obtained by the FEM:

$$
\begin{gathered}
\tau_{f}=c_{i}+\sigma_{n} \tan \varphi_{i}, \\
c_{i}=\left(1-k_{i}\right) c_{r}+k_{i} c_{j}, \\
\tan \varphi_{i}=\left(1-k_{i}\right) \tan \varphi_{r}+k_{i} \tan \varphi_{j}, \\
\sigma_{n}=\frac{\sigma_{x}+\sigma_{y}}{2}+\frac{\sigma_{x}-\sigma_{y}}{2} \cos 2 \alpha-\tau_{x y} \sin 2 \alpha, \\
\tau_{n}=\frac{\sigma_{x}-\sigma_{y}}{2} \sin 2 \alpha+\tau_{x y} \cos 2 \alpha,
\end{gathered}
$$

where $c_{i}$ and $\varphi_{i}$ are the cohesion of a material of $i$ th segment of a slip surface and the friction angle of a material of $i$ th segment of a slip surface, respectively, $c_{r}$ and $\varphi_{r}$ are the cohesion and friction angle of rock bridges, respectively, $c_{i}$ and $\varphi_{i}$ are the cohesion and friction angle of joints, respectively, $\sigma_{n}$ is the stress acting normal to a specified slip surface, $\alpha$ is the angle of normal direction of slip surface to the horizontal plane, $\sigma_{x}, \sigma_{y}$, and $\tau_{x y}$ are the stresses on the slip surface, and $c_{i}$ and $\varphi_{i}$ are the function of the MPR of $i$ th segment of slip surface $k_{i}$.

2.2. The MPR $k$. Figure 1 shows rock bridges in jointed rock masses which are present because of the nonpersistent nature of the joints. In order to calculate the decrease in strength of jointed rock masses in different directions, Wang et al. [14] define the MPR of a rock mass as the ratio of the joint network on the shear failure path when the jointed rock mass is sheared to the damaged state in a certain direction. Figure 2 shows that the MPR $k$ is calculated by

$$
k_{\beta_{0}}=\frac{\sum J L}{\sum J L+\sum R B R},
$$
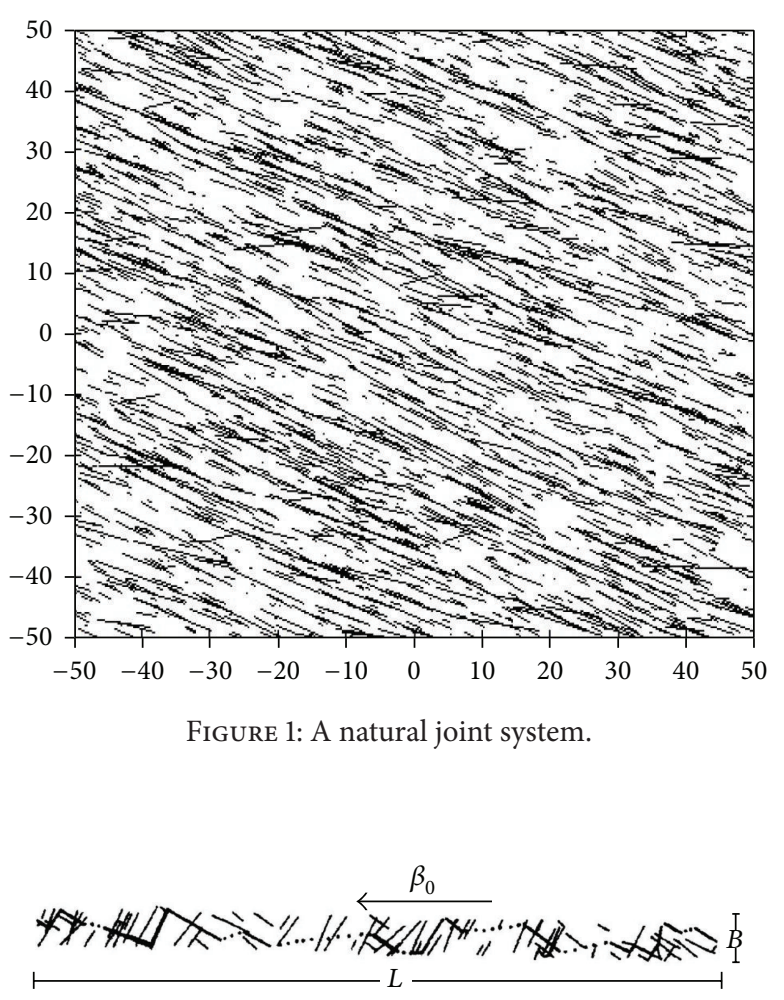

FIgURE 2: The failure path of joints and rock bridges.

where $J L$ and $R B R$ are the projection length of joints and rock bridges in the shear failure path, respectively. As shown in Figure 2, $B$ and $L$ are the projection width and projection length, respectively. $\beta_{0}$ is the visual angle, which can be used to express the direction of the joints.

From (3) and Figure 2, we can observe that the MPR $k$ is related to the projection width $B$ and projection length $L$. Thus the MPR $k$ has the multiscale characteristics.

In the $2 \mathrm{D}$ joint network, there are many combinations of forms of the joints and rock bridges. In order to simplify the calculation, according to the research of $\mathrm{Du}$ et al. [11], it is assumed that there are three basic combinations of the joints and rock bridges, for which the shear resistances are calculated as Figure 3.

The two adjacent joints overlap but do not intersect as shown in Figure 3(a), and the shear resistant force $R$ is expressed as

$$
R=\tau_{j 1} l_{1}+\sigma_{t} h+\tau_{j 2} l_{2}
$$

where $\tau_{j 1}$ and $\tau_{j 2}$ are the sheared strength of the two joints, respectively. $\sigma_{t}$ is tensile strength of the rock bridges. $l_{1}$ and $l_{2}$ are the length of the two joints along the shear plane, respectively. $h$ is the length of the rock bridge along the vertical component of the shear plane and $d$ is the length of the rock bridge along the shear plane. 


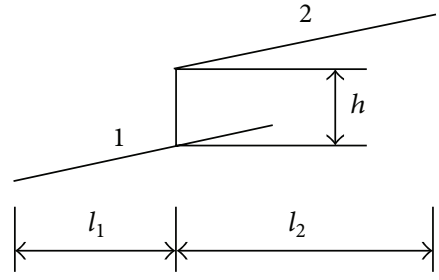

(a)

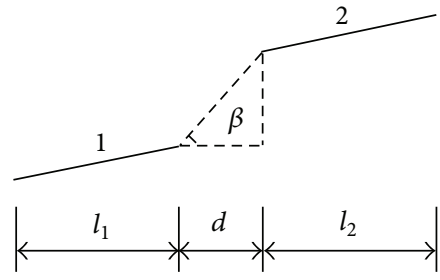

(b)

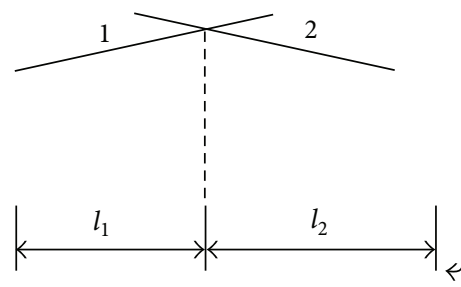

(c)

Figure 3: The three basic combination forms of the joints and rock bridges.

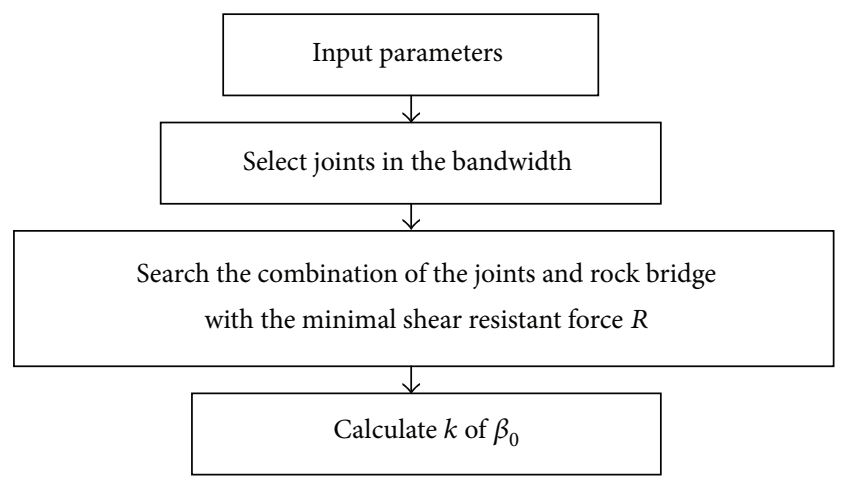

Figure 4: The program flow diagram for calculating $k$.

The two adjacent joints are neither overlapping nor intersecting, as shown in Figure 3(b). The shear resistant force $R$ is expressed as [15]

$$
R= \begin{cases}\tau_{j 1} l_{1}+\sigma_{t} h+\tau_{j 2} l_{2}, & \beta \geq \theta, \\ \tau_{j 1} l_{1}+\tau_{b} h+\tau_{j 2} l_{2}, & \beta<\theta\end{cases}
$$

in which

$$
\begin{gathered}
\tau_{b}=\left[\sigma_{t}\left(\sigma_{t}+\sigma_{n}\right)\right]^{1 / 2}, \\
\theta=\frac{\arctan \left(2 \tau_{b} / \sigma_{n}\right)}{2},
\end{gathered}
$$

where $\tau_{b}$ is the shear strength of the rock bridge along the shear direction, $\theta$ is the angle between the failure plane of the rock bridge and the shear direction, and $\sigma_{n}$ is the average value of the normal stress.

The two adjacent joints are intersecting, as shown in Figure 3(c). The shear resistant force $R$ is expressed as

$$
R=\tau_{j 1} l_{1}+\tau_{j 2} l_{2}
$$

The dynamic programming method is used to search for the failure path of the joints and rock bridges included in the shear bandwidth and then calculate $k$. The coded program flow diagram is shown in Figure 4.

From the aforementioned analysis, we can observe that $k$ has multiscale characteristics. When the bandwidth changes, $k$ also changes.
2.3. The Stress Integration for a Slip Line within an Element. As represented in (1), the line integral of stress along a slip surface is required to calculate the factor of safety. The stress at any point within an element is

$$
\sigma=\sum_{b=1}^{n n} N_{b} \sigma_{b}^{\text {node }}
$$

where $n n$ is the number of nodes in the element, $N_{b}$ is a shape function for a nodal point $b$, and $\sigma_{b}^{\text {node }}$ is the nodal stress.

With simpliication of the theoretical formulations, as shown in Figure 5, only one slip line segment is assumed within an element. And the stress integration for a slip line within an element can be calculated by

$$
\int_{\mathbf{P}_{1}}^{\mathbf{P}_{2}} \sigma d \Gamma=\frac{L}{2} \sum_{i=1}^{n_{\text {int }}} \sigma\left(\mathbf{P}\left(s_{i}\right)\right) W_{i}
$$

in which

$$
\mathbf{P}\left(s_{i}\right)=\frac{\mathbf{P}_{1}+\mathbf{P}_{2}}{2}+\frac{\mathbf{P}_{2}-\mathbf{P}_{1}}{2} s_{i},
$$

where $n_{\text {int }}$ is the number of integration points in the local coordinate system and $s_{i}$ and $W_{i}$ are the local coordinates of the $i$ th integration point and the weight value of the $i$ th integration point, respectively. $\mathbf{P}_{1}$ and $\mathbf{P}_{2}$ are intersection point vectors between the boundary of an element and a slip surface in the global coordinate system, and $L$ is the length between $\mathbf{P}_{1}$ and $\mathbf{P}_{2}$.

When the angle of the slip surface within an element is identified, the $k_{i}$ within an element can be also identified, and (1) can be calculated.

2.4. GA Applied in Locating the CSS. Basically, GA [16] is based on Darwin's theory of survival of the fittest, which is 


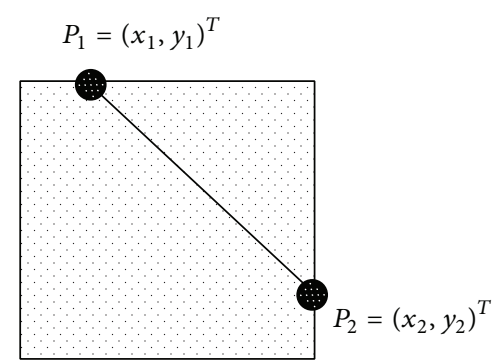

(a) A slip line segment

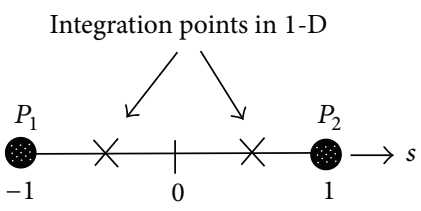

(b) Local coordinate system

FIGURE 5: Slip surface within an element.

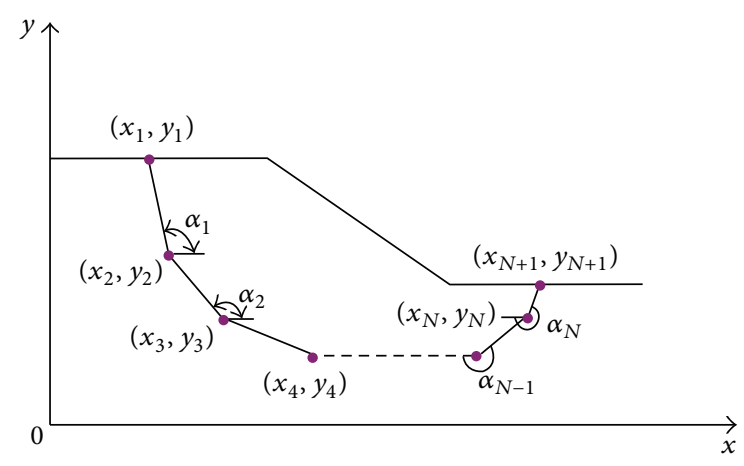

FIGURE 6: The segments of the slip surface.

based on the principle that solutions to a problem can be obtained through evolution. The algorithm starts with a set of possible solutions. The set of possible solutions is called the "population." Each possible solution within the population is called a "chromosome." Associated with each chromosome is a fitness value, which is found by evaluating the chromosome with respect to the objective function. Solutions from one population are taken and used to construct a new population so that the new population (offspring) will be fitter than the old one. This process is repeated until the best chromosome representing the optimum solution is produced or some termination criterion, such as a set number of generations, is reached. The fundamentals of GA are described in the following sections.

2.4.1. Fitness Function. The search for the CSS can be mathematically expressed as the minimization of the factor of safety $F$. And the problem can be written as

$$
\begin{array}{ll}
\min & F \\
\text { subject to } & \alpha_{1} \leq \alpha_{2} \leq \cdots \leq \alpha_{i} \\
& \leq \cdots \leq \alpha_{N}, \quad 1 \leq i \leq N
\end{array}
$$

where $\alpha_{i}$ is the angle of $i$ th segment of the slip surface, which is shown in Figure 6. $N$ is the number of segments making up the slip surface.
The constraint condition can be converted into a penalty function $G\left(\alpha_{1}, \ldots, \alpha_{N}\right)$ and substituted into the objective function, which can be rewritten as

$$
\min \left(F+G\left(\alpha_{1}, \ldots, \alpha_{N}\right)\right)
$$

in which

$$
G\left(\alpha_{1}, \ldots, \alpha_{N}\right)=f_{\text {pun }} F \quad \text { when } \alpha_{i}>\alpha_{i+1},
$$

where $f_{\text {pun }}$ is the penalty factor. And $f_{\text {pun }}$ is adopted as a big value, which is far more than 1 .

2.4.2. Initialization. The GA starts with a population of $N$ possible solutions. Each solution is created by $n$ binary encoding.

2.4.3. Selection. The selection operator is used to determine which chromosomes are chosen as parents that will create offspring for the next generation. In this study, the roulette wheel selection is used in conjunction with elitism. This usually involves retaining the best chromosome at each generation to ensure that the best chromosome is not lost if it is not selected in reproduction or if it is destroyed by crossover or mutation.

2.4.4. Crossover and Mutation. The single-point crossover operator is employed in this study and the randomly flipping mutation procedure is also used.

2.5. The Main Idea of Proposed Model. The program flow diagram for the proposed model is shown in Figure 7.

\section{Numerical Results and Discussions}

3.1. The Numerical Example for Calculating Multiscale Characters of $k$. The distribution of joints was shown in Figure 8. The parameters used in the numerical example were shown in Table 1. The length and depth of model were both $100 \mathrm{~m}$. The average normal stress and the tensile strength of the rock bridges were $2.5 \mathrm{MPa}$ and $2.0 \mathrm{MPa}$, respectively. The sheared strengths of the joints were all 1.95 MPa. The influences of the projection length and projection width on the MPR $k$ were shown in Figures 9 and 10. 


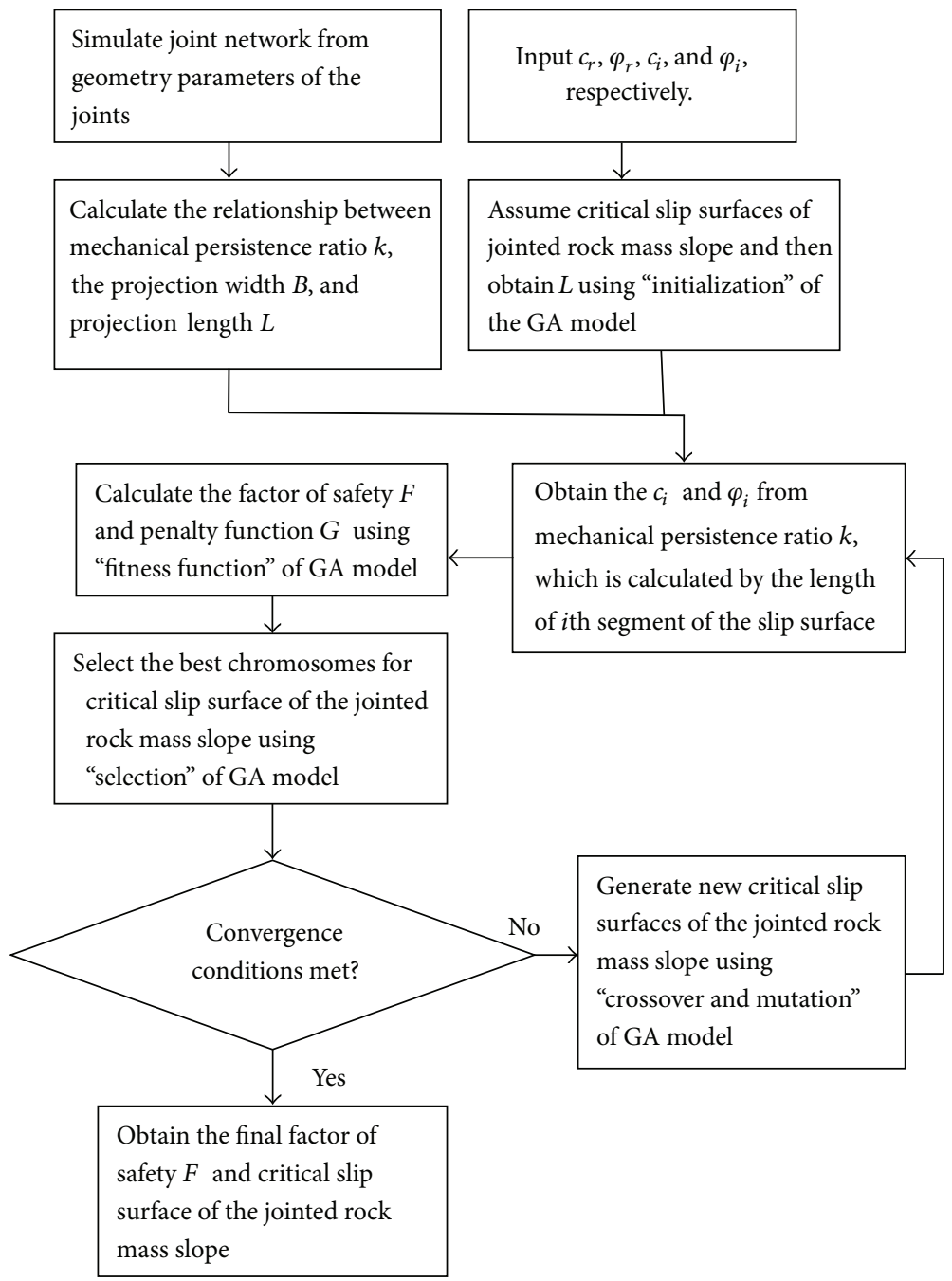

FIgURE 7: The program flow diagram for the proposed model.

TABLE 1: Occurrence geometry parameters of the joints.

\begin{tabular}{|c|c|c|c|c|}
\hline \multirow{2}{*}{ Distribution form } & $\operatorname{Dip}\left({ }^{\circ}\right)$ & Dip angle $\left({ }^{\circ}\right)$ & Spacing $(\mathrm{m})$ & Trace length (m) \\
\hline & Normal & Uniform & Normal & Negative exponential \\
\hline Average value & 129.25 & 28.62 & 2.71 & 3.7 \\
\hline Standard deviation & 36.88 & 11.29 & 6.03 & 0.58 \\
\hline
\end{tabular}

Example 1 was used to describe the multiscale characteristics of the MPR $k$. Through observing the results in Figure 8 to Figure 10, they showed that the MPR $k$ was related to the projection width $B$ and projection length $L$. Thus the MPR $k$ had the multiscale characteristics. Thus when calculating the CSSs of JRMS, it was necessary to consider the relationship between MPR $k$ and the length of each segment of the slip surface.

3.2. Numerical Example: A Homogeneous Slope. This example was a homogeneous slope. The geometry and FE meshes of the slope were shown in Figures 11 and 12, respectively. The coefficient of cohesion $c$ and friction angle $\varphi$ of the slope were $9.8 \mathrm{KPa}$ and $10^{\circ}$, respectively. The elastic modulus $E$ and Poisson ratio $\nu$ of the slope were $200 \mathrm{MPa}$ and 0.3 , respectively. The density of the slope $\rho$ was $1764 \mathrm{~kg} / \mathrm{m}^{3}$. The distribution of von Mises stress in the slope was shown in Figure 13. The CSS of slope was shown in Figure 14. The factor of safety of slope $F=1.3228$. And other results of the CSS and factor of safety of slope were shown in Figure 14 and Table 2.

Example 2 was given for verifying the proposed model. The slope was homogeneous. And the results calculated by proposed model were compared with those calculated by other models in related literatures. In Figure 14, Kang et al. used an artificial bee colony algorithm (ABC) to locate the CSS of slopes and the factor of safety $F$ was calculated by the 
TABLE 2: Statistics of the results of Example 2.

\begin{tabular}{lcccccc}
\hline F calculated by Kang et al. [17] & $F$ calculated by Sun et al. [4] & $F$ calculated by proposed model & Min. & Max. & Mean. & SD. \\
\hline 1.3206 & 1.3210 & 1.3228 & 1.3206 & 1.3228 & 1.3215 & $1.172 \times 10^{-3}$ \\
\hline
\end{tabular}

Min.: minimum value; Max.: maximum value; Mean.: mean value; SD.: standard deviation.

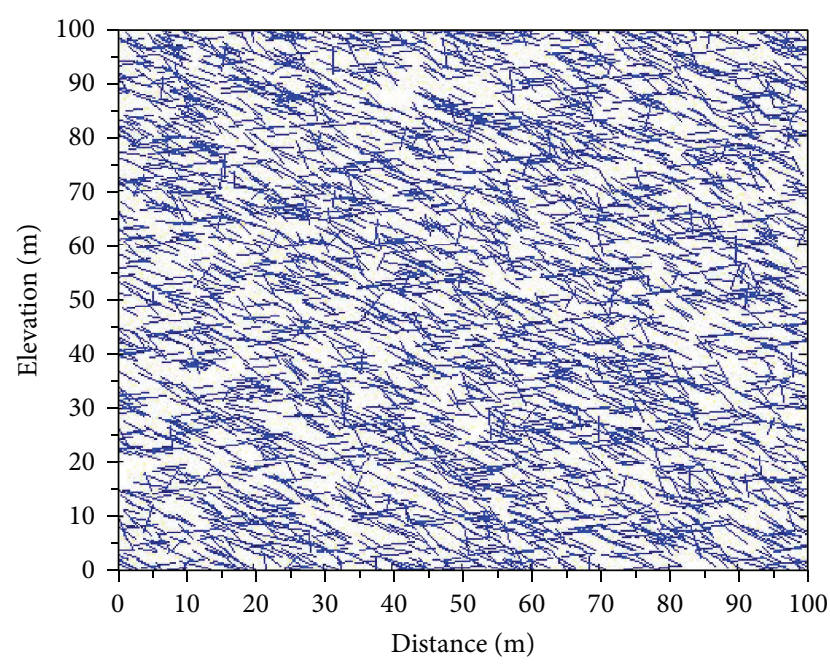

FIGURE 8: The distribution of joints.

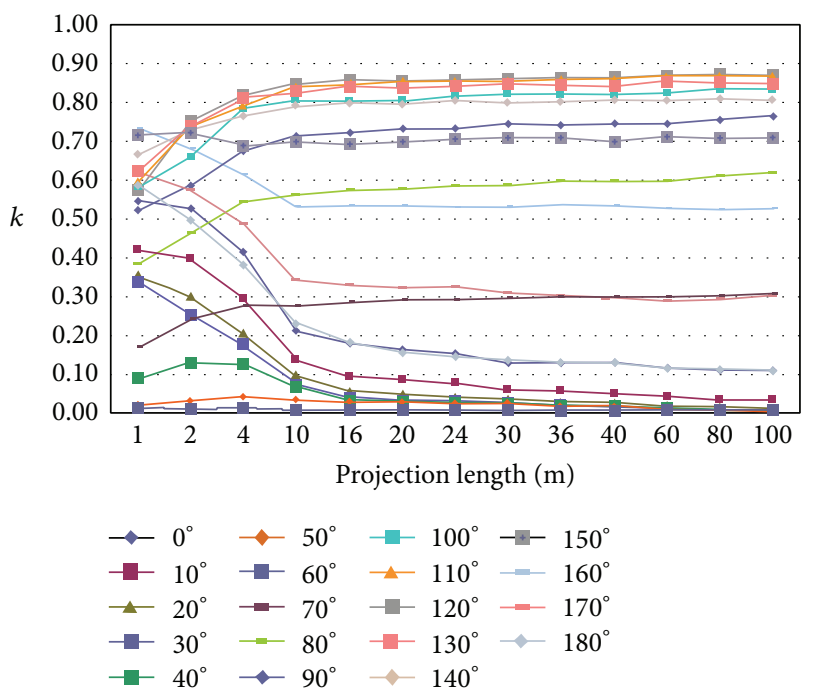

FIGURE 9: The influence of the projection length on the MPR $k(\mathrm{~m})$.

Spencer method. Sun et al. used a Spline-based GA to locate the CSS of slopes.

Through observing the results shown in Figure 11 to Figure 14 and Table 2, they showed that the mean value, minimum value, and maximum value of $F$ are 1.3215, 1.3206 and 1.3228 , respectively. The standard deviation of the factor of safety $F$ of the slope calculated by the proposed model and those used elsewhere was $0.1172 \%$. And the value of $F$ calculated by proposed model based on GA was greater than that calculated by the model based on ABC and the model based on Spline-based GA. The reason was mainly that the

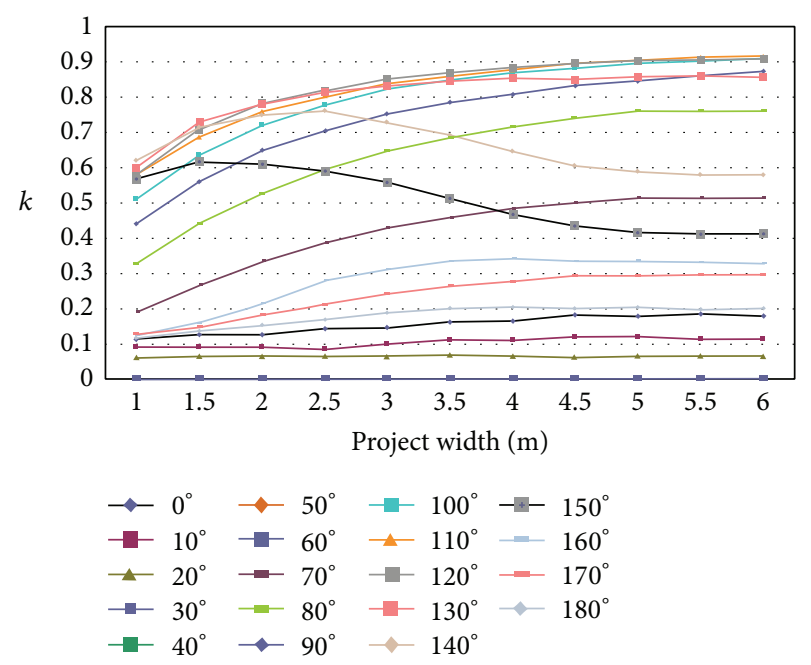

FIGURE 10: The influence of the projection width on the MPR $k(\mathrm{~m})$.

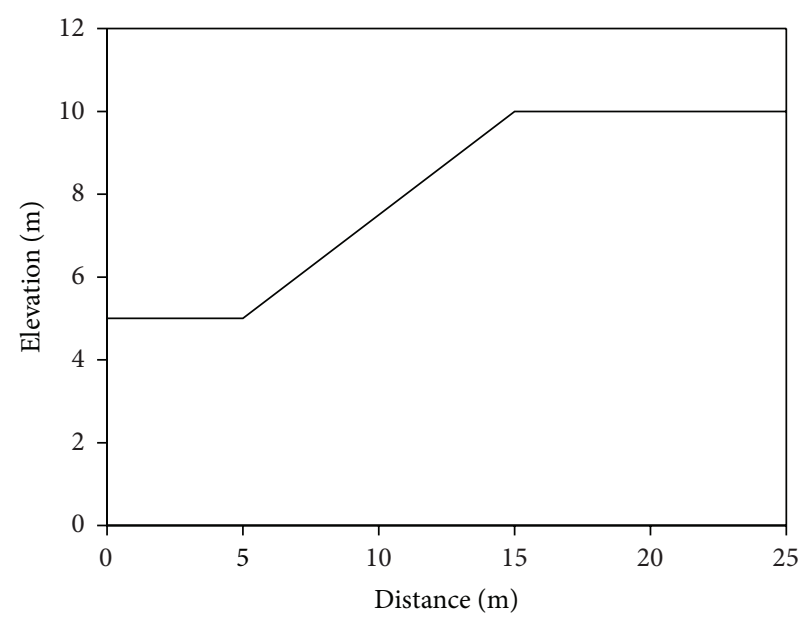

FIGURE 11: The geometry of the slope.

methods to calculate the factor of safety $F$ and to locate the CSS of slopes are different. The proposed model used the FEM to analyze the stress field near the CSS, which considered the effects of the deformation on the stress in the slope. However, the values of $F$ calculated by different models were close. These results showed that the proposed model could calculate the factor of safety $F$ and the CSS of homogeneous slope well.

3.3. Numerical Example: A Homogeneous Layered Slope. This example was a homogeneous layered slope. The geometry and FE meshes of the slope were shown in Figures 15 and 16 , respectively. The physical parameters for the layered slope were shown in Table 3. The distribution of von Mises stress 


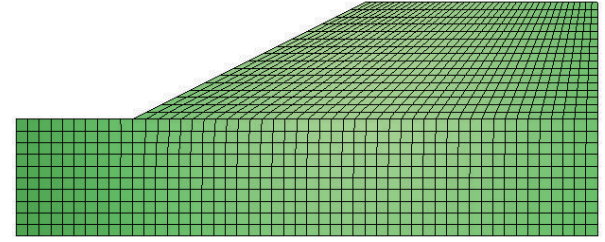

FIGURE 12: FE meshes of the slope.

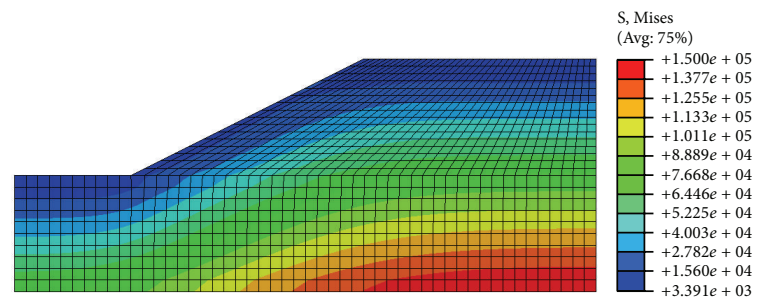

FIGURE 13: The distribution of von Mises stress of slope.

TABLE 3: Physical parameters for layered slope.

\begin{tabular}{lccccc}
\hline Layers & $c(\mathrm{KPa})$ & $\varphi\left({ }^{\circ}\right)$ & $\rho\left(\mathrm{kg} / \mathrm{m}^{3}\right)$ & $E(\mathrm{MPa})$ & $\nu$ \\
\hline 1 & 15.0 & 20.0 & 1900 & 200 & 0.3 \\
2 & 17.0 & 21.0 & 1900 & 200 & 0.3 \\
3 & 5.0 & 10.0 & 1900 & 200 & 0.3 \\
4 & 35.0 & 28.0 & 1900 & 200 & 0.3 \\
\hline
\end{tabular}

in the slope was shown in Figure 17. The CSS of the slope was shown in Figure 18. The factor of safety of slope $F=1.3418$. And other results of the CSS and factor of safety of slope were shown in Figure 18 and Table 4.

Example 3 was also given for verifying the proposed model. This example was a homogeneous layered slope. And the results calculated by the proposed model were compared with those calculated by other models in related literatures. In Figure 19, Kang et al. used an artificial bee colony algorithm (ABC) to locate the CSS of slopes and the factor of safety $F$ was calculated by the Spencer method. Li et al. used a realcoded genetic algorithm (RCGA) to locate the CSS of slopes.

Through observing the results of Figure 15 to Figure 18 and Table 4, they showed that the mean value, minimum value, and maximum value of $F$ were 1.3232, 1.2917, and 1.3418, respectively. The standard deviation of the factor of safety $F$ of the homogeneous layered slope calculated by the proposed model and those used elsewhere was $2.740 \%$. And the value of $F$ calculated by proposed model was also greater than that calculated by the model based on ABC and the model based on RCGA. The reason was mainly that the methods to calculate the factor of safety $F$ of the CSS of the slope were different. The proposed model used the FEM to analyze the stress in the layered slope, which considered the effects of the deformation on the stress in the layered slope. However, the values of $F$ calculated by different models were close. These results showed the proposed model could calculate the factor of safety $F$ and the CSS of layered slope well.

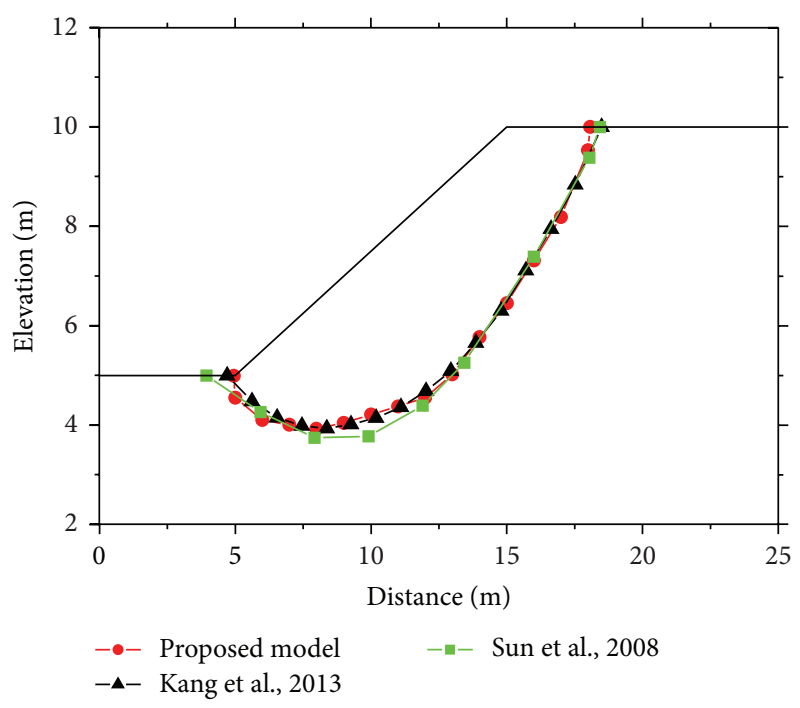

FIGURE 14: The CSSs for the slope $[4,17]$.

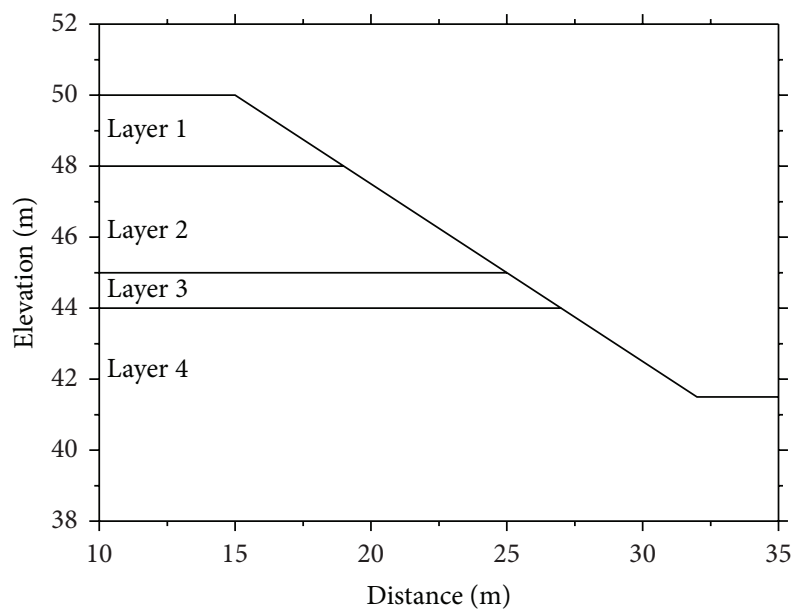

FIGURE 15: The geometry of the layered slope.

\subsection{Numerical Example: Homogeneous Layered Earth Rockfill} Dam. This example was a homogeneous layered earth rockfill dam. The geometry and FE meshes of the earth rockfill dam were shown in Figures 19 and 20, respectively. The physical parameters for the layered earth rockfill dam were shown in Table 5. The distribution of von Mises stress in the earth rockfill dam was shown in Figure 21. The CSS for the earth rockfill dam was shown in Figure 22. The factor of safety of the earth rockfill dam $F=1.3868$. And other results of the CSS and factor of safety of earth rockfill dam were shown in Figure 22 and Table 6.

Example 4 was also given for verifying the proposed model. This example was a homogeneous layered slope. And the results calculated by proposed model were compared with those calculated by other models in related literatures. In Figure 22, Kang et al. used an artificial bee colony algorithm $(\mathrm{ABC})$ to locate the CSS of slopes and the factor of safety 
TABLE 4: Statistics of the results of Example 3.

\begin{tabular}{lcccccc}
\hline$F$ calculated by Kang et al. [17] & $F$ calculated by Li et al. [6] & $F$ calculated by proposed model & Min. & Max. & Mean. & SD. \\
\hline 1.2917 & 1.3360 & 1.3418 & 1.2917 & 1.3418 & 1.3232 & $2.740 \times 10^{-2}$ \\
\hline
\end{tabular}

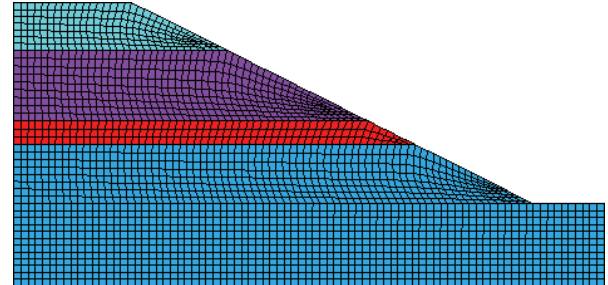

FIGURE 16: FE mesh of the layered slope.

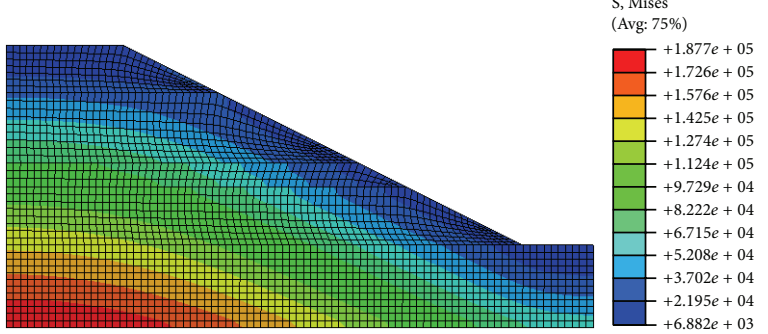

FIGURE 17: The distribution of von Mises stress of layered slope.

TABle 5: Physical parameters for layered slope.

\begin{tabular}{lccccc}
\hline Layers & $c(\mathrm{KPa})$ & $\varphi\left({ }^{\circ}\right)$ & $\rho\left(\mathrm{kg} / \mathrm{m}^{3}\right)$ & $E(\mathrm{MPa})$ & $\nu$ \\
\hline 1 & 49.0 & 29.0 & 2038 & 200 & 0.3 \\
2 & 0.0 & 30.0 & 1764 & 200 & 0.3 \\
3 & 7.84 & 20.0 & 2038 & 200 & 0.3 \\
4 & 0.0 & 30.0 & 1764 & 200 & 0.3 \\
\hline
\end{tabular}

$F$ was calculated by the Spencer method. Sun et al. used a Spline-based GA to locate the CSS of slopes.

Through observing the results of Figure 19 to Figure 22 and Table 6 , they showed that the mean value, minimum value, and maximum value of $F$ were $1.3865,1.3776$, and 1.3950 , respectively. The standard deviation of the factor of safety $F$ of the earth rockfill dam calculated by the proposed model and those used elsewhere was $0.8705 \%$. And the value of $F$ calculated by proposed model was more close to the mean value. The reason was mainly that the methods to calculate the factor of safety $F$ of the CSS of the earth rockfill dam were different. The proposed model used the FEM to analyze the stress in the earth rockfill dam, which considered the effects of the deformation on the stress in the earth rockfill dam. However, the values of $F$ calculated by different models were close. These results showed that the proposed model could calculate the factor of safety $F$ and the CSS of the earth rockfill dam well.

3.5. Numerical Example: JRMS. This example was a JRMS. The geometry and FE meshes of the slope were the same as

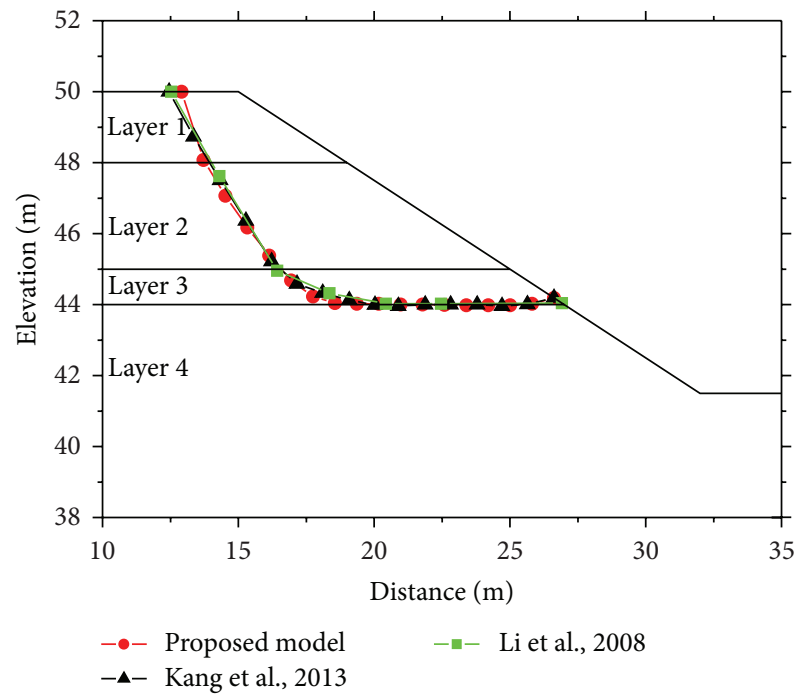

FIGURE 18: The CSSs for a layered slope $[6,17]$.

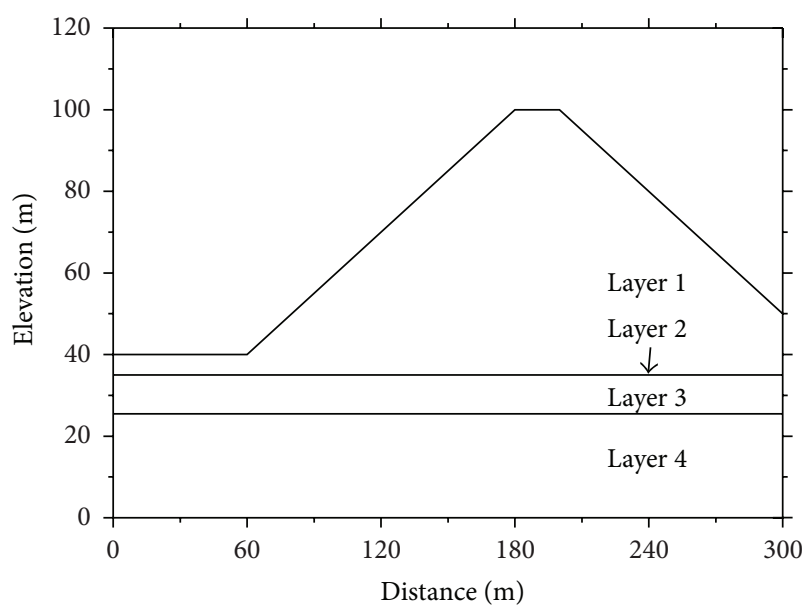

FIGURE 19: The geometry of the earth rockfill dam.

those used in Example 2. The coefficient of cohesion $c$ and friction angle $\varphi$ of the rock bridges in the slope were $9.8 \mathrm{KPa}$ and $10^{\circ}$, respectively, which were the same as those used in Example 2. The coefficients of cohesion $c$ and friction angle $\varphi$ of the joints of slope were $3.0 \mathrm{KPa}$ and $7^{\circ}$, respectively. The elastic modulus $E$, Poisson ratio $\nu$, and density $\rho$ of the slope were the same as those used in Example 2. Thus, the distribution of von Mises stress of slope was the same as that in Example 2. The MPR $k$ using different projection lengths was shown in Figure 23. The CSS of JRMS was shown in Figure 24. And the results of the CSS and factor of safety of JRMS were shown in Figure 24 and Table 7. 
TABLE 6: Statistics of the results of Example 4.

\begin{tabular}{lcccccc}
\hline F calculated by Kang et al. [17] & $F$ calculated by Sun et al. [4] & $F$ calculated by proposed model & Min. & Max. & Mean. & SD. \\
\hline 1.3776 & 1.3950 & 1.3868 & 1.3776 & 1.3950 & 1.3865 & $8.705 \times 10^{-3}$
\end{tabular}

TABLE 7: Statistics of the results of Example 5.

\begin{tabular}{lcc}
\hline$F$ for homogeneous slope & $F$ for JRMS in projection length $=100 \mathrm{~m}$ & $F$ for JRMS in multiscale projection length \\
\hline 1.3228 & 1.255 & 1.302
\end{tabular}

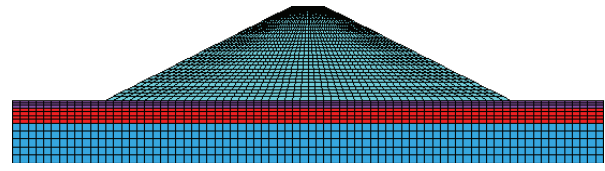

FIgURE 20: FE mesh of the earth rockfill dam.

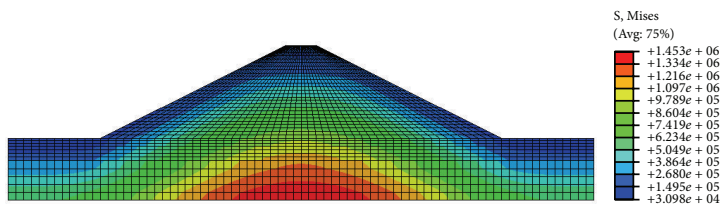

FIGURE 21: The distribution of von Mises stress in the earth rockfill dam.

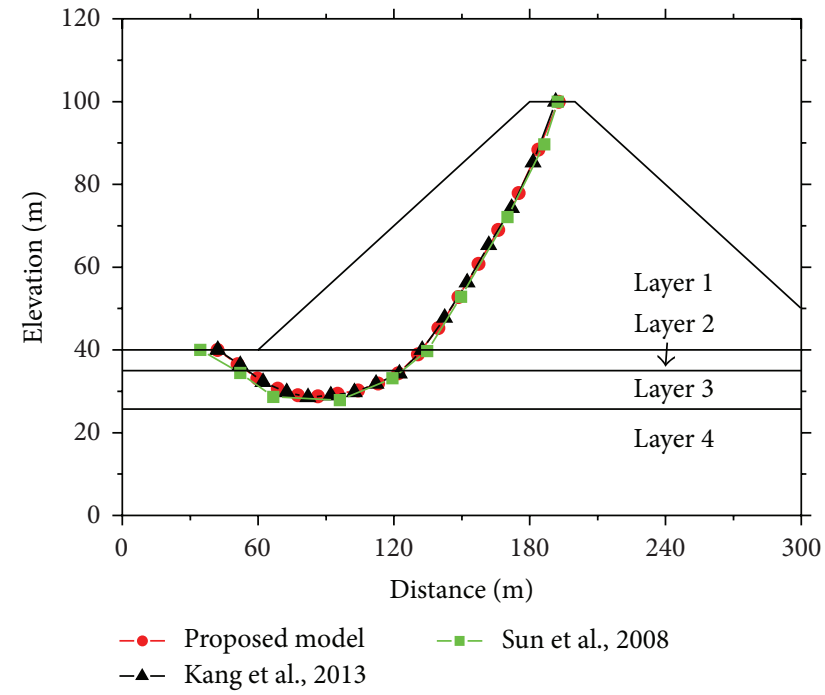

FIgURE 22: The CSSs for the earth rockfill dam $[4,17]$.

Example 5 was used to analyze a JRMS. This example firstly used the model, which did not consider the multiscale characteristics of the MPR $k$. In other words, the MPR $k$ did not change when the length of any segment of the slip surface was different. Then the material parameters of joints and rock bridges such as cohesion and friction angle remained constant. Then this example used the model, which considered the multiscale characteristics of the MPR $k$. In other words, the MPR $k$ changed when the length of segment of slip surface was different. Then the material parameters of

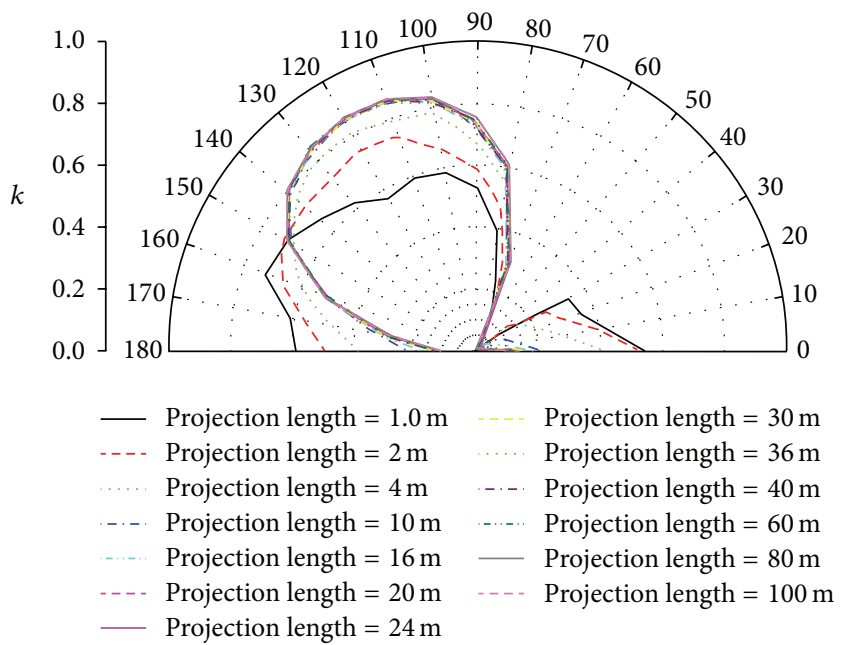

FIgURE 23: The rose diagram of $k$ using a multiscale projection length.

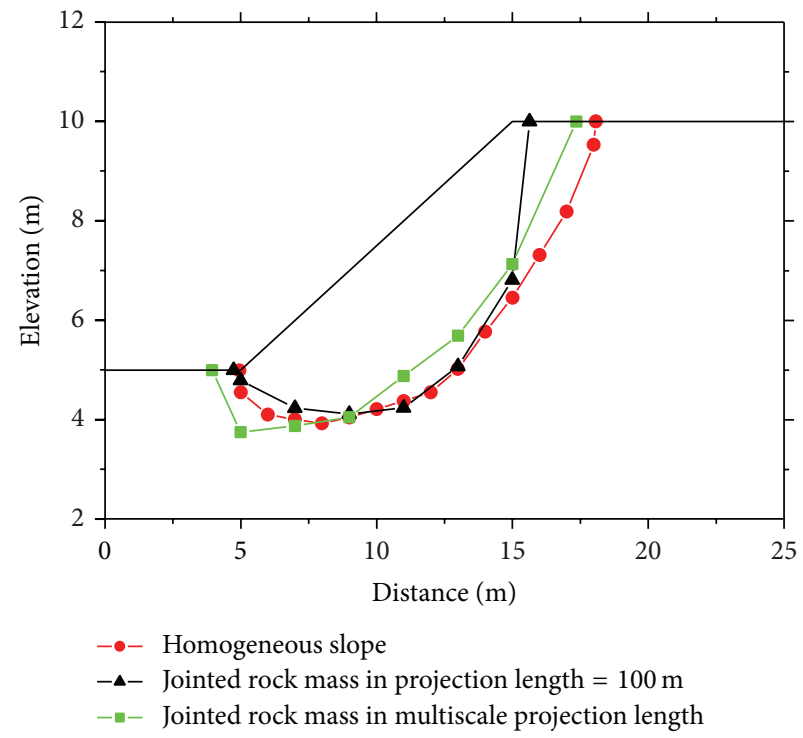

FIGURE 24: The CSS of the JRMS.

joints and rock bridges such as cohesion and friction angle also changed. And through observing the results of Figure 24 and Table 7, they showed that the multiscale characteristics of the MPR $k$ had great influence on the CSS and factor of safety of JRMS. The factors of safety of JRMS calculated by 
different models were both less than that of homogeneous slope. And if the distribution of the MPR $k$ changed much in different projection length, the CSS and factor of safety of JRMS would vary significantly. The factor of safety of the JRMS as calculated by the proposed model, which considered the multiscale characteristics of the MPR, was 3.75\% more than that calculated by the proposed model, which did not consider the multiscale characteristics of the MPR. It showed that the multiscale characteristics of the MPR played an important role in the stability of the JRMS.

\section{Conclusion}

A model based on a GA for locating the multiscale CSSs in a statistical JRMS from the stress field was proposed. The proposed model could consider the influence of different sets of joints on the material strength of a jointed rock mass and the multiscale characteristics of the MPR $k$. The results showed that the MPR was related to the projection width and projection length. Thus the MPR had the multiscale characteristics and these played an important role in locating the CSS in a JRMS. The results also showed that the proposed model could calculate CSSs and factors of safety of slopes accurately.

\section{Conflict of Interests}

The authors declare that there is no conflict of interests regarding the publication of this paper.

\section{Acknowledgments}

This study was supported by the National Natural Science Foundation of China (Grant nos. 51109029, 51178081, 51138001, and 51009020) and the State Key Development Program for Basic Research of China (no. 2013CB035905).

\section{References}

[1] A. T. C. Goh, "Search for critical slip circle using genetic algorithms," Civil Engineering and Environmental Systems, vol. 17, no. 3, pp. 181-211, 2000.

[2] P. Mccombie and P. Wilkinson, "The use of the simple genetic algorithm in finding the critical factor of safety in slope stability analysis," Computers and Geotechnics, vol. 29, no. 8, pp. 699-714, 2002.

[3] A. R. Zolfaghari, A. C. Heath, and P. F. McCombie, "Simple genetic algorithm search for critical non-circular failure surface in slope stability analysis," Computers and Geotechnics, vol. 32, no. 3, pp. 139-152, 2005.

[4] J. Sun, J. Li, and Q. Liu, "Search for critical slip surface in slope stability analysis by spline-based GA method," Journal of Geotechnical and Geoenvironmental Engineering, vol. 134, no. 2, pp. 252-256, 2008.

[5] A. Sengupta and A. Upadhyay, "Locating the critical failure surface in a slope stability analysis by genetic algorithm," Applied Soft Computing Journal, vol. 9, no. 1, pp. 387-392, 2009.

[6] Y.-C. Li, Y.-M. Chen, T. L. T. Zhan, D.-S. Ling, and P. J. Cleall, "An efficient approach for locating the critical slip surface in slope stability analyses using a realcoded genetic algorithm," Canadian Geotechnical Journal, vol. 47, no. 7, pp. 806-820, 2010.

[7] J. Y. Kim and S. R. Lee, "An improved search strategy for the critical slip surface using finite element stress fields," Computers and Geotechnics, vol. 21, no. 4, pp. 295-313, 1997.

[8] S. E. Cho and S. R. Lee, "Instability of unsaturated soil slopes due to infiltration," Computers and Geotechnics, vol. 28, no. 3, pp. 185-208, 2001.

[9] C. H. Wang, X. Y. Xia, and G. Li, "Genetic algorithm for searching for critical slip surface in soil slopes based on stress fields," Journal of Tsinghua University, vol. 44, no. 3, pp. 425428, 2004.

[10] H. Zheng, G. H. Sun, and D. Liu, "A practical procedure for searching critical slip surfaces of slopes based on the strength reduction technique," Computers and Geotechnics, vol. 36, no. 1-2, pp. 1-5, 2009.

[11] J. C. Du, Z. Y. Chen, H. L. Mi, X. G. Wang, and J. C. Zhou, "Determination of comprehensive shear strength for jointed rock masses in 3D condition using genetic algorithm and Monte-Carlo method," Chinese Journal of Rock Mechanics and Engineering, vol. 23, no. 13, pp. 2157-2163, 2004.

[12] J. P. Chen, B. Lu, X. M. Gu, J. H. Fan, and Y. H. Cheng, "Numerical simulation study on 3D comprehensive shear strength of fractured rockmass," Chinese Journal of Rock Mechanics and Engineering, vol. 25, no. 7, pp. 1463-1468, 2006.

[13] W. Zhang, J. P. Chen, and Q. Wang, "Determination of the most dangerous sliding surface for fractured rock mass slope based on fracture frequency," Journal of Engineering Geology, no. 20, pp. 692-696, 2012.

[14] X. G. Wang, Z. Y. Chen, and W. S. Liu, "Determination of joint persistence and shear strength parameters of rock masses by Monte-Carlo method," Chinese Journal of Rock Mechanics and Engineering, vol. 11, no. 4, pp. 345-355, 1992.

[15] L. E. Lajtai, "Strength of discontinuous rocks in direct shear," Geotechnique, vol. 19, no. 2, pp. 218-2, 1969.

[16] A. T. C. Goh, "Genetic algorithm search for critical slip surface in multiple-wedge stability analysis," Canadian Geotechnical Journal, vol. 36, no. 2, pp. 382-391, 1999.

[17] F. Kang, J. J. Li, and Z. Y. Ma, "An artificial bee colony algorithm for locating the critical slip surface in slope stability analysis," Engineering Optimization, vol. 45, no. 2, pp. 207-223, 2013. 


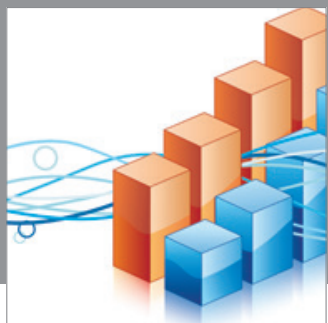

Advances in

Operations Research

mansans

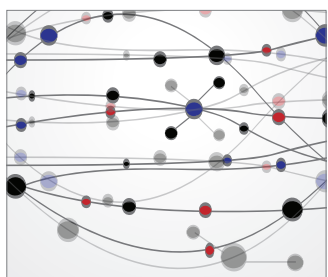

The Scientific World Journal
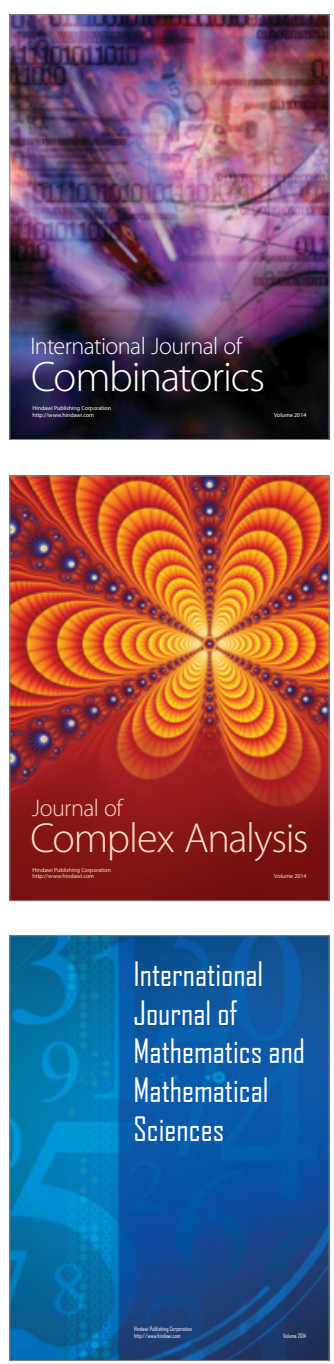
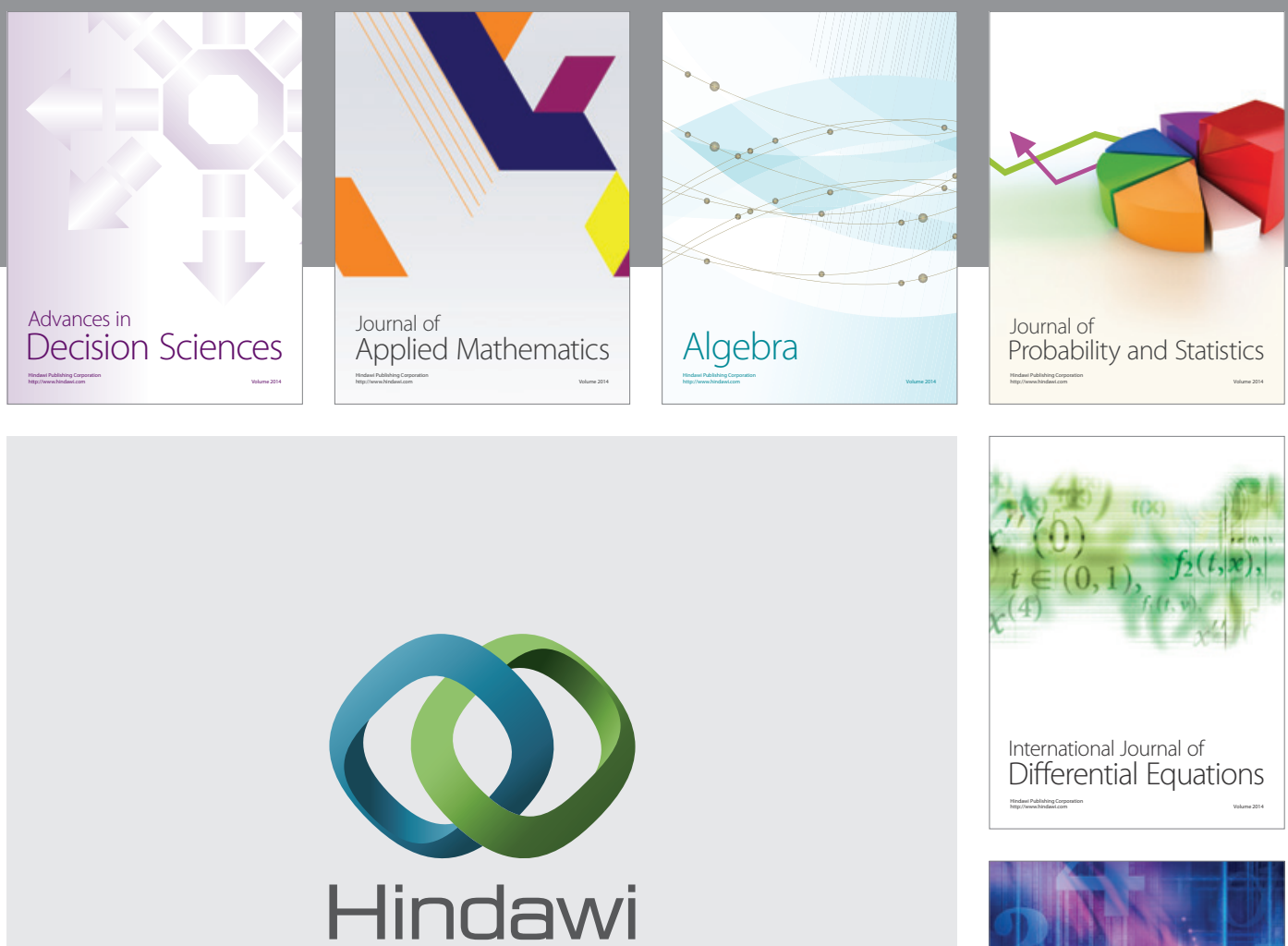

Submit your manuscripts at http://www.hindawi.com
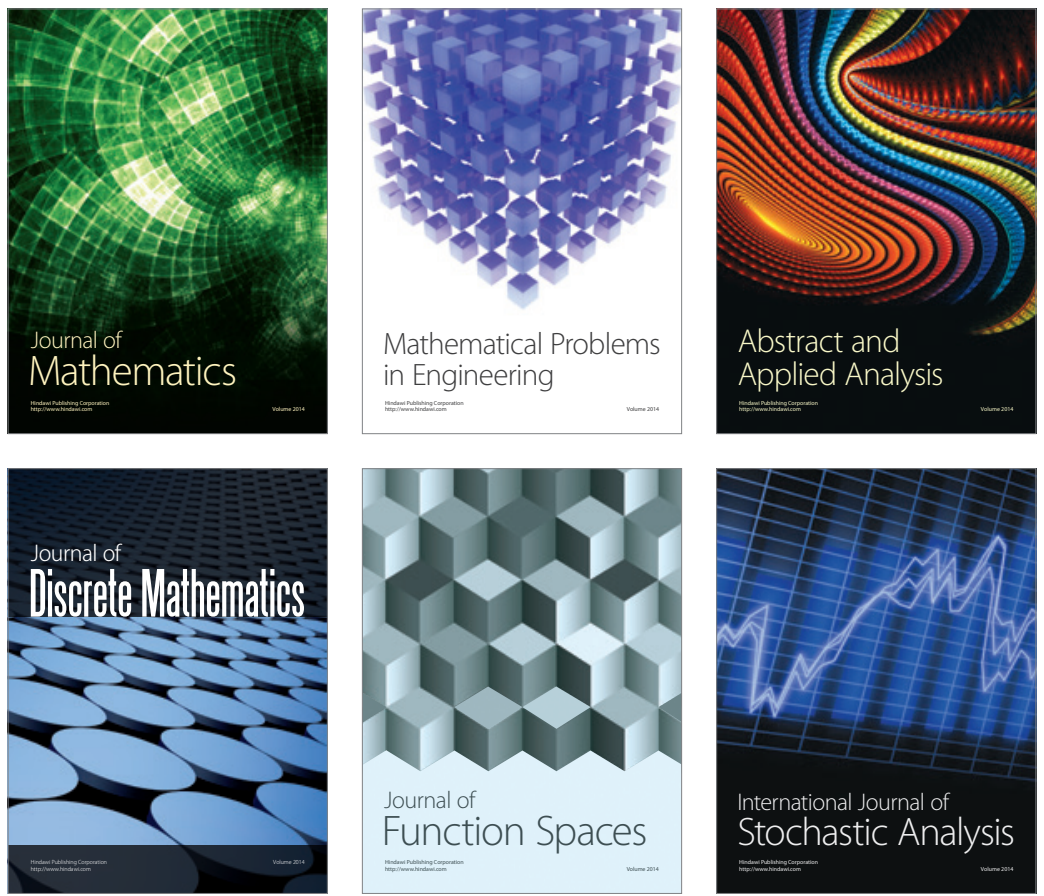

Journal of

Function Spaces

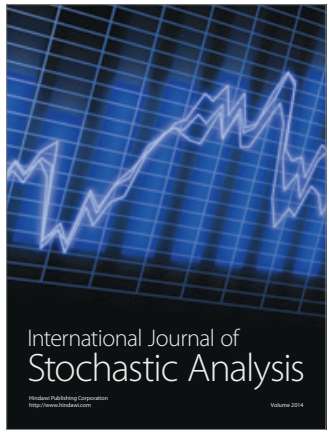

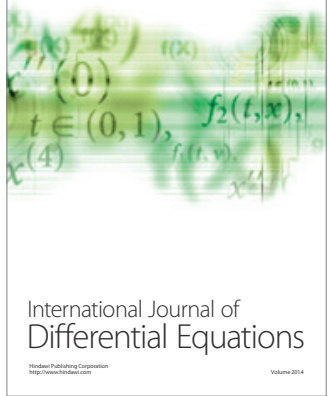
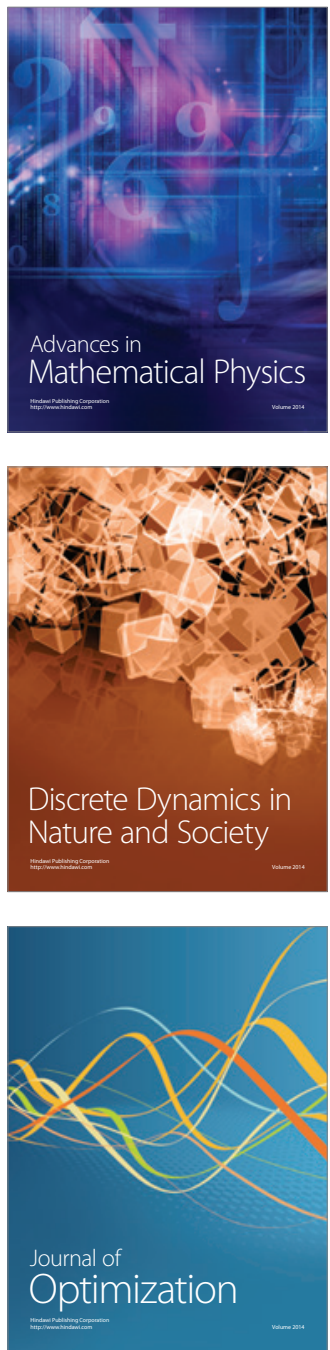